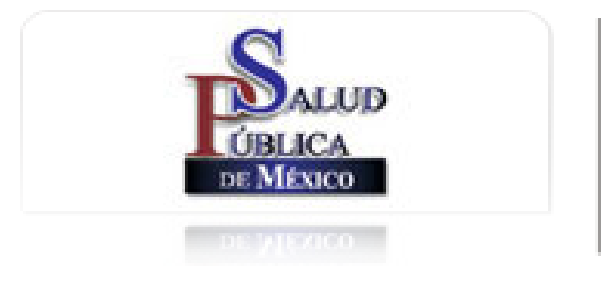

Salud Pública de México

ISSN: 0036-3634

spm@insp.mx

Instituto Nacional de Salud Pública

México

Reyes-Morales, Hortensia; Flores-Hernández, Sergio; Sauceda-Valenzuela, Alma Lucila; VértizRamírez, José de Jesús; Juárez-Ramírez, Clara; Wirtz, Veronika J; Pérez-Cuevas, Ricardo Percepción de los usuarios sobre la calidad de la atención ambulatoria en servicios de salud en México

Salud Pública de México, vol. 55, núm. 2, 2013, pp. S100-S105 Instituto Nacional de Salud Pública

Cuernavaca, México

Disponible en: http://www.redalyc.org/articulo.oa?id=10628331005

Cómo citar el artículo

- Número completo

- Más información del artículo

- Página de la revista en redalyc.org

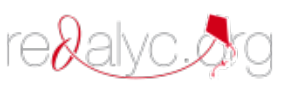

Sistema de Información Científica

Red de Revistas Científicas de América Latina, el Caribe, España y Portugal Proyecto académico sin fines de lucro, desarrollado bajo la iniciativa de acceso abierto 


\title{
Percepción de los usuarios sobre la calidad de la atención ambulatoria en servicios de salud en México
}

\begin{abstract}
Hortensia Reyes-Morales, D en C,(1) Sergio Flores-Hernández, M en C,(2) Alma Lucila Sauceda-Valenzuela, M en C,(1) José de Jesús Vértiz-Ramírez, M en C,(I) Clara Juárez-Ramírez, D en C,(I) Veronika J Wirtz, PhD,(I)
\end{abstract}

Ricardo Pérez-Cuevas, D en C. (3)

\section{Reyes-Morales H, Flores-Hernández S, Sauceda-Valenzuela AL, Vértiz-Ramírez JJ, Juárez-Ramírez C, Wirtz VJ, Pérez-Cuevas R. \\ Percepción de los usuarios sobre la calidad de la atención ambulatoria en servicios de salud en México. Salud Publica Mex 2013;55 supl 2:S100-S105.}

\section{Resumen}

Objetivo. Identificar la percepción de los usuarios de los servicios de salud ambulatorios en México sobre la calidad de atención y los factores asociados con dicha percepción. Material y métodos. De los datos obtenidos por las Encuestas Nacionales de Salud y Nutrición 2006 y 2012 se analizó la percepción de la atención recibida y de mejoría en salud después de la atención; se elaboró un modelo multivariado para identificar los factores de los servicios asociados con la percepción de mejoría. Resultados. La buena percepción de atención se elevó de 82 a $85 \%$ y y la de mejoría del estado de salud, de 79 a $81 \%$, entre los años 2006 y 2012. La explicación sobre la enfermedad y tratamiento, la consulta sin cita previa, la oportunidad de elegir el servicio de salud y menor tiempo de espera se asociaron con la percepción de mejoría. Conclusiones. Existen avances en la calidad de atención percibida en la atención ambulatoria en México. Es necesario fortalecer estrategias organizacionales para brindar atención con mayor oportunidad y responder a las expectativas de los usuarios.

Palabras clave: percepción; calidad; atención primaria de salud; encuestas; México
Reyes-Morales H, Flores-Hernández S,

Sauceda-Valenzuela AL, Vértiz-Ramírez JJ,

Juárez-Ramírez C, Wirtz VJ, Pérez-Cuevas R.

Users' perception about quality of ambulatory

healthcare services in Mexico.

Salud Publica Mex 2013;55 suppl 2:S100-S105.

\begin{abstract}
Objective. To identify users' perception about ambulatory healthcare services quality and associated factors in Mexico. Materials and methods. Analysis of the 2006 and 2012 National Surveys of Health and Nutrition that included users' perception of healthcare services and improvement in health status. A multivariate regression analysis allowed identifying the associated factors with the perception of improved health status. Results. Between 2006 and 2012, users' positive perception of healthcare services increased from 82 to $85 \%$, and user report of improvement in health status increased from 79 to $81 \%$. Health status improvement, explanations about the disease and treatment, being attended without appointment, freedom to choose the provider and short waiting time were associated with a perception of better quality of care. Conclusions. Users' perception about the quality of care in ambulatory healthcare settings has improved, yet it is still neccessary to strengthen organizational strategies to provide healthcare when needed and to improve fulfillment of users' expectations.
\end{abstract}

Key words: perception; quality; primary care; surveys; Mexico

(I) Centro de Investigación en Sistemas de Salud, Instituto Nacional de Salud Pública. Cuernavaca, Morelos, México.

(2) Dirección General, Instituto Nacional de Salud Pública. Cuernavaca, Morelos, México.

(3) Banco Interamericano de Desarrollo. Washington, DC.

Fecha de recibido: 7 de diciembre de 2012 - Fecha de aceptado: 7 de enero de 2013 Autor de correspondencia: Dra. Hortensia Reyes-Morales. Centro de Investigación en Sistemas de Salud, Instituto Nacional de Salud Pública. Av. Universidad 655, Col. Santa María Ahuacatitlán. 62100 Cuernavaca, Morelos, México.

Correo electrónico: hortensia.reyes@insp.mx 
$\mathrm{U}$ na de las funciones principales de los sistemas de salud es la provisión de servicios equitativos, oportunos, efectivos y seguros, en los que la calidad óptima de la atención es un elemento fundamental para garantizarlos. ${ }^{1}$ Desde hace más de dos décadas, el concepto de calidad de atención ha incluido el enfoque tanto individual como poblacional, asegurando el otorgamiento de servicios de salud con base en la mejor evidencia científica disponible y con énfasis en los resultados deseables en salud. ${ }^{2}$ Este concepto implica que los pacientes estén informados y compartan la toma de decisiones para su atención, aunque tradicionalmente el análisis de la calidad de atención se ha centrado en la dimensión técnica u objetiva, más que en la dimensión desde la perspectiva del paciente, la cual se considera subjetiva. $^{3}$

Recientemente se ha hecho énfasis en el desarrollo de diferentes métodos aplicados y validados en poblaciones y organizaciones específicas para medir la calidad, como la aplicación de encuestas ${ }^{4-7}$ y escalas;, 89 asimismo, se han analizado los conceptos de evaluación de esta dimensión desde diferentes perspectivas. ${ }^{10-12}$

En México, la opinión de los usuarios de los servicios de salud sobre la calidad de los mismos ha sido estudiada principalmente por medio de las encuestas nacionales de salud, ${ }^{13,14}$ de encuestas en regiones específicas ${ }^{15} \mathrm{y}$ evaluaciones de programas nacionales. ${ }^{16,17} \mathrm{El}$ seguimiento de los resultados con indicadores similares a través del tiempo es fundamental para evaluar los cambios en la provisión de los servicios y garantizar una atención ambulatoria de calidad, en un sistema de salud cuya magnitud se sustenta en los casi doscientos millones de consultas médicas que se otorgan anualmente en el país, principalmente en las instituciones públicas, pero en el que el sector privado tiene también gran importancia en aproximadamente $40 \%$ de las atenciones ambulatorias. $^{18}$

Por lo anterior, el objetivo principal del presente análisis fue identificar, a partir de la Encuesta Nacional de Salud y Nutrición (ENSANUT) 2012, la percepción de los usuarios sobre la calidad de atención y la mejoría en su salud después de dicha atención; posteriormente, la percepción resultante se contrastó con la reportada en la ENSANUT 2006. Como objetivo secundario, se exploraron los factores que influyeron en la percepción de la mejoría de los utilizadores de los servicios en 2012.

\section{Material y métodos}

Se efectuó un análisis secundario de la ENSANUT 2012 a partir de datos del cuestionario de utilizadores, cuya fuente de información fue la entrevista directa a 14104 personas que solicitaron y recibieron servicios ambulatorios de salud, en las dos semanas previas a la entrevista, por enfermedad, lesión, accidente, rehabilitación, servicio dental, programa de control de diabetes o hipertensión arterial.

De manera resumida, la ENSANUT 2012 es una encuesta probabilística nacional de México, con representatividad a nivel de los estados, por estratos nacionales urbano y rural. La información se obtuvo mediante un procedimiento de muestreo polietápico hasta nivel de hogar; para el apartado de utilizadores, en cada uno de ellos se seleccionó a una o dos personas que cumplieron esta condición (solicitar y recibir algún servicio ambulatorio de salud). ${ }^{18}$ Para identificar la calidad de la atención, en el presente análisis se incluyó información de las siguientes variables contenidas en el cuestionario de utilizadores:

1) Percepción de la atención recibida. La pregunta realizada fue: El servicio que le dio la persona que lo (la) atendió, ¿le pareció...? (opciones de respuesta: muy bueno, bueno, regular, malo, muy malo).

2) Resultados en salud (percepción de mejoría después de la atención). A partir de la pregunta: Después de la última atención que recibió, considera que su estado de salud... (opciones de respuesta: mejoró mucho, mejoró, no cambió, empeoró, empeoró mucho).

En todos los usuarios se identificaron las razones para calificar la atención recibida y en las personas que expresaron que no regresarían a solicitar atención en el mismo lugar se registraron las razones de la decisión.

El sitio de atención se registró de acuerdo con la siguiente clasificación: Secretaría de Salud (SSa), Instituto Mexicano del Seguro Social (IMSS); servicios privados que incluyen consultorios privados y consultorios dependientes de farmacias, y otras instituciones: Petróleos Mexicanos (Pemex), Secretaría de la Defensa Nacional (Sedena), Secretaría de Marina (Semar), Programa IMSS-Oportunidades, Instituto de Seguridad y Servicios Sociales para los Trabajadores del Estado (ISSSTE, ISSSTE estatal). Tanto la SSa como el Programa IMSS-Oportunidades ofrecen atención a población que carece de seguridad social y que puede o no estar afiliada al Seguro Popular (seguro público de salud para esta población).

Para evaluar la asociación con la percepción de mejoría, se analizó el tiempo de evolución del episodio por el que acudió a consulta; el sitio de atención que otorgó la atención; tipo de personal de salud (médico general o especialista, dentista, enfermera, nutriólogo, personal comunitario, acupunturista, homeópata, dependiente de farmacia); si tuvo oportunidad de elegir el lugar de atención; si la consulta fue programada mediante cita; 
tiempo de espera para la atención; tiempo de duración de la consulta; si recibió explicaciones sobre la enfermedad y el tratamiento; si pagó por la atención recibida, y si obtuvo los medicamentos prescritos en el mismo sitio de la consulta.

\section{Análisis estadístico}

Las dos variables de calidad de atención se analizaron en dos categorías: para la percepción de la atención recibida se clasificó como buena y mala percepción (a partir de las opciones de respuesta servicio muy bueno o bueno vs. regular, malo, o muy malo); para la variable percepción de mejoría después de la atención, las categorías fueron mejoró o no mejoró (de las opciones de respuesta mejoró mucho o mejoró vs. no cambió, empeoró, empeoró mucho). De todas las variables se obtuvieron frecuencias absolutas y relativas. Posteriormente se contrastaron las proporciones de las variables de calidad de atención con las de la ENSANUT $2006,{ }^{19}$ por sitio de atención.

La asociación cruda entre la percepción de mejoría posterior a la atención recibida y las variables independientes se midió mediante el análisis bivariado con estimación de razón de momios cruda $\left(R M_{c}\right)$ e intervalos de confianza al 95\% (IC95\%). Finalmente, se determinaron los factores asociados con la percepción de mejoría posterior a la atención mediante un modelo de regresión logística para obtener las razones de momios ajustadas $\left(\mathrm{RM}_{\mathrm{a}}\right)$ y errores estándar. El modelo fue ajustado por edad, sexo, condición de ruralidad y tiempo de evolución de la enfermedad. Se evaluó la calibración del modelo mediante la prueba de bondad del ajuste para encuestas complejas. El análisis se realizó con el programa estadístico STATA versión 12.0 (Stata, Stata Corp, College Station, TX).

\section{Resultados}

Los 14104 utilizadores que se incluyeron en el análisis representan a una población de 10162076 personas que recibieron atención ambulatoria en los 15 días previos a la entrevista. La atención fue otorgada, en su mayoría, por médicos generales $(78.2 \%)$ y, en menor proporción, por médicos especialistas $(16.8 \%)$ u otro tipo de personal $(5.0 \%)$. Más de la mitad de los usuarios reportaron haber obtenido atención en un servicio correspondiente a su institución de afiliación, y 34.6\% de las consultas fue programada.

La proporción de usuarios que en 2006 y 2012 reportaron buena percepción del servicio de salud se presenta en la figura 1, y la proporción de mejoría del estado de salud posterior a la atención, en la figura 2. La buena percepción se elevó de 82\% en 2006 a 85\% en

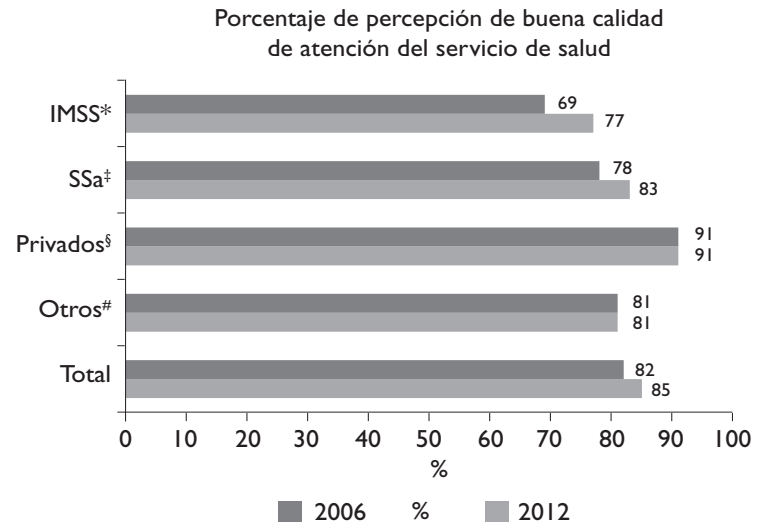

* Instituto Mexicano del Seguro Social

¥ Centro de salud u hospital de la Secretaría de Salud

$\S$ Médicos privados; consultorios dependientes de farmacias

\# Otros: Pemex, Sedena, Semar, IMSS-Oportunidades, ISSSTE, ISSSTE

Estatal, otro lugar

Figura I. Porcentaje de percepción de buena calidad DE ATENCIÓN DEL SERVICIO DE SALUD, POR TIPO DE SERVICIO. MÉxIco, 2006-2012

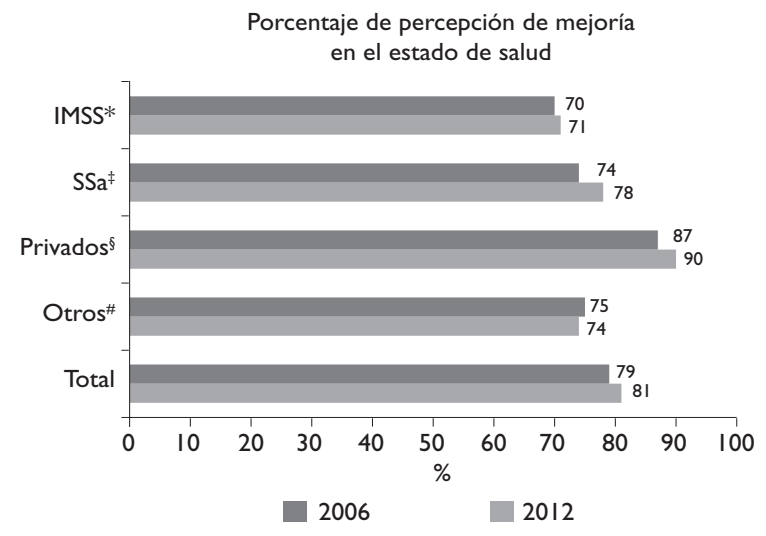

* Instituto Mexicano del Seguro Social

¥ Centro de salud u hospital de la Secretaría de Salud

$\S$ Médicos privados; consultorios dependientes de farmacias

\# Otros: Pemex, Sedena, Semar, IMSS-Oportunidades, ISSSTE, ISSSTE

Estatal, otro lugar

Figura 2. Percepción de mejoría en el estado de salud POSTERIOR a LA ATENCIÓN, POR tiPo de SERVICIO. MÉXICO, 2006-20I2

2012, y la percepción de mejoría del estado de salud posterior a la atención se incrementó de 79 a $81 \%$ en el mismo periodo. Al analizar por sitio de atención, se observó que la SSa y el IMSS aumentaron en 5 y 8\%, 
respectivamente, la buena percepción de sus servicios, aunque el IMSS tuvo el porcentaje más bajo en ambos periodos respecto a los demás servicios. La atención en servicios privados tuvo la mejor percepción, sin cambio entre los dos periodos; la percepción de mejoría del estado de salud aumentó de 87 a 90\%. El resto de los servicios mantuvo una aceptable proporción de buena percepción sin modificación entre los dos años analizados.

En 2012, los principales motivos de la buena percepción fueron buena atención (44.3\%), buen trato (39.9\%), explicación del médico acerca de la enfermedad y su tratamiento $(15 \%)$ y la prontitud en la atención $(10.7 \%)$. En contraste, los motivos expresados por quienes no regresarían fueron el tiempo de espera prolongado $(28.5 \%)$, mal trato $(23.6 \%)$, falta de mejoría $(21 \%)$, desacuerdo con el diagnóstico o el tratamiento $(20.8 \%)$ y falta de medicamentos (15.9\%) (datos no mostrados en tablas).

Los factores de los servicios asociados con la percepción de mejoría en el estado de salud fueron haber recibido explicaciones sobre la enfermedad $\left(\mathrm{RM}_{\mathrm{a}}=2.77\right.$; IC95\% 2.16-3.55); haber recibido explicaciones sobre el tratamiento $\left(\mathrm{RM}_{\mathrm{a}}=1.91\right.$; IC95\% 1.47-2.82); haber obtenido consulta sin cita previa $\left(\mathrm{RM}_{\mathrm{a}}=1.75\right.$; IC95\% 1.462.08); haber tenido la oportunidad de elegir el servicio de salud $\left(\mathrm{RM}_{\mathrm{a}}=1.35\right.$; IC95\% 1.14-1.67), y que el tiempo de espera para la consulta fuera menor a 30 minutos $\left(\mathrm{RM}_{\mathrm{a}}=1.29\right.$; IC95\% 1.08-1.55) (cuadro I y figura 3).

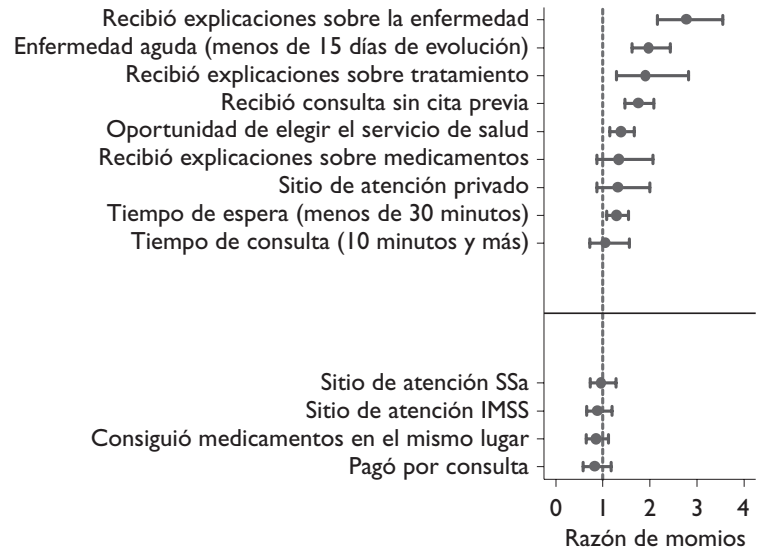

Figura 3. Factores asociados CON PERCEPCIÓN de mejoría POSTERIOR A LA ATENCIÓN EN SERVICIOS AMBULATORIOS DE Salud. Modelo de regresión logística** México, 2012

\section{Discusión}

Desde la Encuesta Nacional de Salud II (ENSA II), la proporción de los usuarios que reporta buena calidad se ha mantenido en niveles aproximados a $80 \% .{ }^{13}$ Este estudio destaca el incremento de la buena percepción de la calidad de atención entre 2006 y 2012 para los servicios de salud otorgados por las principales insti-

Cuadro I

FACTORES ASOCIAdOS CON PERCEPCIÓN de MEJORÍA POSTERIOR A LA ATENCIÓN EN SERVICIOS AMBULATORIOS DE SALUd. Modelo de regresión logística,* MÉxico, 2012

Variable

Razón de momios ajustada Error estándar Valor de $p \quad$ Intervalo de confianza 95\%

\begin{tabular}{|c|c|c|c|c|}
\hline Recibió explicaciones sobre la enfermedad & 2.77 & 0.351 & $<0.001$ & $2.16-3.55$ \\
\hline Recibió explicaciones sobre tratamiento & 1.91 & 0.379 & 0.001 & I.29-2.82 \\
\hline Recibió consulta sin cita previa & 1.75 & 0.157 & $<0.001$ & I.47-2.08 \\
\hline Oportunidad de elegir el servicio de salud & 1.38 & 0.131 & $<0.001$ & $1.15-1.67$ \\
\hline Tiempo de espera menor a 30 minutos & 1.29 & 0.210 & $<0.01$ & I.07-1.55 \\
\hline Recibió explicaciones sobre medicamentos & 1.34 & 0.296 & NS & $0.87-2.06$ \\
\hline Atención en servicios privados & 1.32 & 0.278 & NS & $0.87-1.99$ \\
\hline Duración de la consulta (10 minutos o más) & 1.06 & 0.210 & NS & $0.78-1.56$ \\
\hline Atención en la SSa & 0.97 & 0.137 & NS & $0.73-1.28$ \\
\hline Atención en el IMSS & 0.89 & 0.134 & NS & $0.66-1.19$ \\
\hline Consiguió los medicamentos en el mismo sitio de atención & 0.85 & 0.120 & NS & $0.64-1.12$ \\
\hline Pagó por la consulta & 0.82 & 0.148 & NS & $0.58-1.76$ \\
\hline
\end{tabular}

* Modelo ajustado por edad, sexo, condición de ruralidad y tiempo de evolución de la enfermedad

NS No significativo 
tuciones públicas: IMSS y SSa. Esta variación podría ser resultado de la política en salud para la mejora de la calidad integrada, a partir del año 2000, de acuerdo con las estrategias y acciones llevadas a cabo bajo la Cruzada Nacional por la Calidad de los Servicios de Salud y el Sistema Integral de Calidad en Salud (SICALIDAD). ${ }^{20}$ Esta política se reforzó en 2006; un ejemplo son las guías de práctica clínica sectoriales (GPC), como apoyo para el otorgamiento de atención con base en evidencia y como criterios objetivos de desempeño para la evaluación rutinaria de la atención en los servicios de salud. ${ }^{21}$ Aunque la mayor elevación en el porcentaje de buena percepción de calidad en el IMSS podría explicarse por la amplia difusión e implementación de las GPC en esta institución, ${ }^{22}$ con la consecuente mejora en la calidad tanto técnica como interpersonal estos cambios no se observan en la percepción de mejoría en el estado de salud posterior a la consulta, que resulta mejor para 2012 en la SSa y los servicios privados.

Una explicación a la falta de consistencia entre los dos indicadores analizados es el tipo diferente de población que atiende cada institución: la del IMSS está más envejecida ( $11.4 \%$ con más de 65 años de edad ${ }^{23}$ que la población general de México $(6.18 \%$ para este mismo grupo de edad) ${ }_{,}^{24}$ con mayor demanda de atención por padecimientos crónicos, cuyas condiciones clínicas repercuten menos en la mejoría percibida.

Resulta interesante que dos de los factores con mayor importancia incluidos en el modelo estén relacionados con la explicación sobre las condiciones de la enfermedad y el tratamiento indicado. Esto refleja la relevancia que tiene la comunicación del médico u otro personal de salud para la satisfacción de las personas atendidas en los servicios ambulatorios, lo que coincide con otros reportes. ${ }^{11}$ La relación interpersonal como atributo central de la continuidad de la atención en los servicios ambulatorios requiere del conocimiento mutuo que genera confianza; sin embargo, la complejidad actual de los sistemas de salud con sobrecargas de consulta en sus servicios, poca integración de equipos de salud, rotación del personal y restricciones de la población para un acceso permanente a un mismo proveedor son barreras para una atención satisfactoria. ${ }^{25,26}$

Otras variables significativas estuvieron relacionadas con la organización de los servicios. Debe considerarse la importancia que los usuarios otorgan a la facilidad de acceso, la oportunidad de elección del servicio y el menor tiempo de espera. Este aspecto queda manifiesto en la mejor percepción de los servicios privados encontrada tanto en la ENSANUT 2012 como en estudios previos. $16,17,27$

Con el reconocimiento de que una atención integradora, equitativa y centrada en las personas requiere tomar en cuenta las preferencias, necesidades y valores de los usuarios potenciales para guiar las decisiones y acciones de los proveedores de servicios de salud, la percepción de los pacientes ha adquirido cada vez mayor relevancia. ${ }^{28}$ Paralelamente, el avance social que ha permitido mayor participación de la población en la definición de las políticas públicas ha ubicado el derecho a un acceso a servicios de salud de calidad como prioridad en la que las necesidades y expectativas poblacionales obligan a una respuesta adecuada por parte del propio sistema de salud. ${ }^{29,30}$

En conclusión, si bien la mejora en la percepción de los servicios públicos de salud es relevante, aún es necesario fortalecer la capacidad de respuesta de los servicios públicos de salud. Es recomendable incorporar estrategias organizativas para brindar atención con mayor oportunidad; asimismo, se requiere consolidar los programas de educación continua del personal de salud en atención primaria. Una estrategia particularmente importante es el fortalecimiento de los indicadores de calidad técnica e interpersonal con el fin de mantener una evaluación permanente y detectar áreas de mejora para su corrección oportuna.

\section{Agradecimientos}

Los autores agradecen los valiosos comentarios del doctor Mauricio Hernández a este trabajo.

Declaración de conflicto de intereses. Los autores declararon no tener conflicto de intereses.

\section{Referencias}

I. Thomson S, Osborn R, Squires D, Jun M, eds. International profiles of Health Care Systems, 2012 [monografía en internet]. The Commonwealth Fund 2012. [Consultado 2012 nov 30]. Disponible en: http:// www.commonwealthfund.org/ /media/Files/Publications/Fund\%20 Report/2012/Nov/I645_Squires_intl_profiles_hlt_care_systems_2012.pdf. 2. Lohr KN, Schroeder SA. A strategy for quality assurance in Medicare. N Engl J Med 1990;322:707-7|2.

3. Thierlscher $\mathrm{C}$. How to find the right approach to quality measurement: determinants of quality and its measurement in healthcare. World Medical \& Health Policy 20I0;2(I):375-385.

4. Lim MK, Yang H, Zhang T, Feng W, Zhou Z. Public perceptions of private Health care in socialist China. Health Aff 2004;23(6):222-234. 5. Al-Hoqail IA, Abdalla AM, Saeed AA, Al-Hamdan NA, Bahnassy AA. Pilgrims satisfaction with ambulatory Health services in Makkah, 2008. J Fam Comm Med 2010;17(3): I35-140.

6. Lin H, Xirasagar S, Laditka J. Patient perceptions of service quality in group versus solo practice clinics. Int $\int$ Qual Health Care 2004; 16(6):437-445.

7. Kumar Patro B, Kumar R, Goswami A, Nongkynrih B, Pandav Ch, UG Study Group. Community perception and client satisfaction about primary health care services in an urban resettlement colony of New Delhi. Indian J Comm Med 2008;33(4):250-254. 
8. Cabrera-Arana GA, Bello-Parías LD, Londoño-Pimienta JL. Calidad percibida por usuarios de hospitales del programa de Reestructuración de Redes de Servicios de Salud de Colombia. Rev Salud Publica 2008;10(4):593-604.

9. Haddad S, Potvin L, Roberge D, Pineault R, Remondin M. Patient perception of quality following a visit to a doctor in a primary care unit. Fam Pract 2000; 17: 21-29.

10. Losada-Otálora M, Rodríguez-Orejuela A. Calidad del servicio de salud: una revisión a la literatura desde la perspectiva del marketing. Cuad Adm Bogotá 2007;20(34):237-258.

II. Sohi S, Hole AR, Mead N, McDonald R, Whalley D, Bower P, et al. What patients want from primary care consultations: A discrete choice experiment to identify patients' priorities. Ann Fam Med 2008;6: 107-I I 5. 12. Ridd M, Shaw A, Lewis G, Salisbury C. The patient-doctor relationship: A synthesis of the qualitative literature on patients' perspectives. $\mathrm{Br}$ J Gen Pract 2009;59:268-275.

I3. Ramírez-Sánchez TJ, Nájera-Aguilar P, Nigenda-López G. Percepción de la calidad de la atención de los servicios de salud en México: perspectiva de los usuarios. Salud Publica Mex 1998;40:3-12.

14. Puig A, Pagán JA, Wong R. Assesing quality across health care subsystems in Mexico. J Ambul Care Manage 2009;32(2):I23-I31.

15. Ortiz Espinosa RM, Muñoz Juárez S, Lechuga Martín del Campo D,

Torres Carreño E. Consulta externa en instituciones de salud de Hidalgo, México, según la opinión de los usuarios. Rev Panam Salud Publica 2003; 12(4):229-238.

16. Nigenda-López G. Juárez C, Ruiz JA, Duarte MB, Bonilla P, Aguilar E, et al. Evaluación de la Cruzada Nacional por la Calidad de los Servicios de Salud, 2006-2007. Cuernavaca: Secretaría de Salud/ Instituto Nacional de Salud Pública, 2008

17. González-Block MA, Nigenda G, Téllez M. Trejo B, González LM, Herrera C, et al. Evaluación del Sistema de Protección Social en Salud 2007. Resumen ejecutivo. Cuernavaca: Secretaría de Salud/ Instituto Nacional de Salud Pública, 2008.

18. Gutiérrez JP, Rivera-Dommarco J, Shamah-Levy T, Villalpando-

Hernández S, Franco A, Cuevas-Nasu L, et al. Encuesta Nacional de Salud y Nutrición 2012. Resultados nacionales. Cuernavaca: Instituto Nacional de Salud Publica, 2012.

19. Olaiz-Fernández G, Rivera-Dommarco ], Shamah-Levy T, Rojas R, Villalpando-Hernández S, Hernández-Avila M, et al. Encuesta Nacional de Salud y Nutrición 2006. Cuernavaca: Instituto Nacional de Salud Pública, 2006. 20. Secretaría de Salud. Sistema Integral de Calidad (SICALIDAD) [sitio de internet]. México: Secretaría de Salud, 2008. [Consultado 2012 dic 8]. Disponible en: http://www.calidad.salud.gob.mx/doctos/calidad/pa_sicalidad.pdf.
21. Secretaría de Salud. Programa de Acción Específico 2007-2012. Desarrollo de guías de práctica clínica [sitio de internet]. México: Secretaría de Salud, 2008. [Consultado: 2012 dic 8]. Disponible en: http://www.cenetec. gob.mx/interior/gaceta l0/desarrollo_gpc.html.

22. González-Block MA, López-Santibáñez C, Aguilera-Aburto N, Aldama A, Barajas ME, Becerril Montekio V, et al. Evaluación y estrategias de portabilidad y convergencia hacia la integración del Sistema Nacional de Salud. Resumen ejecutivo. Cuernavaca: Instituto Nacional de Salud Pública, 2011.

23. Instituto Mexicano del Seguro Social. Población derechohabiente adscrita a médico familiar por grupo de edad, 200I-20II. Entorno demográfico y epidemiológico y otros factores de presión sobre el gasto médico [sitio de internet]. México: Instituto Mexicano del Seguro Social, 2012. [Consultado 20I2 dic 9]. Disponible en: www.imss.gob.mx/estadisticas/Documents/201 12012/C02.pdf.

24. Instituto Nacional de Estadística y Geografía. Distribución por edad y sexo. Población total según grupos de edad y sexo por países seleccionados, 2010 [sitio de internet]. México: INEGI, 20I0. [Consultado 2012 dic 9]. Disponible en: http://www.inegi.org.mx/sistemas/sisept/Default. aspx? $\mathrm{t}=$ mdemo $86 \& \mathrm{~s}=\mathrm{est} \& \mathrm{c}=\mid 7498$.

25. Kearley KE, Freeman GF, Heath A. An exploration of the value of the personal doctor-patient relationship in general practice. $\mathrm{Br}$ J Gen Pract 2001;51:712-718

26. Fan VS, Burman M, McDonell MB, Fihn SD. Continuity of care and other determinants of patient satisfaction with primary care. J Gen Intern Med 2005;20:226-233.

27. Sauceda-Valenzuela AL, Wirte VJ, Santa-Ana-Téllez Y, Kageyama-Escobedo ML. Ambulatory health services user's experience of waiting time and expediture and factors associated with the perception of low quality of care in Mexico: BMC Health Serv Res 2010;10: I78. doi:I0.1 I86/14726963-10-178.

28. Institute of Medicine (IOM). Crossing the quality chasm: A new Health System for the 2 I st Century (200I). Report brief [sitio de internet]. Washington: IOM, 200I. [Consultado 2012 diciembre I]. Disponible en: http://books.n ap.edu/html/quality_chasm/reportbrief.pdf 29. Stephens JH, Ledlow GR. Real healthcare reform: focus on primary care Access. Hosp Top 2010;88(4):98-106.

30. Knaul FM, González-Pier E, Gómez-Dantés O, García-Junco D, Arreola-Ornelas $\mathrm{H}$, Barraza-Lloréns $\mathrm{M}$, et al. The quest for universal health coverage: achieving social protection for all in Mexico. Lancet 2012 oct 6;380(9849):I259-I279. doi: 10.1016/S0|40-6736(I2)61068-X 